\title{
High-efficiency conjugated-polymer-hosted blue phosphorescent light-emitting diodes
}

\author{
NIU QiaoLi*, ZHANG Yong, WANG YongLi, WANG Xin \& HE Miao \\ Institute of Optoelectronic Materials and Technology, South China Normal University, LED Industrial Service Platform of Guangzhou City, \\ Guangzhou 510631, China
}

Received June 10, 2011; accepted July 6, 2011; published online May 30, 2012

\begin{abstract}
Highly-efficient blue phosphorescent light-emitting diodes were fabricated based on a conjugated-polymer host by doping bis(2-(4,6-difluorophenyl)-pyridinato-N,C2') picolinate (FIrpic) into poly(9,9-dioctylfluorene) (PFO). Previously, conjugated polymers were not considered as potential hosts for blue phosphorescent dyes because of their low-lying triplet energy levels. Energy back transfer would occur and lead to poor luminescent efficiency in both photoluminescence (PL) and electroluminescence (EL) processes. However, by inserting a hole-transporting layer of poly $(N$-vinylcarbazole) (PVK), the energy back transfer was suppressed. At low FIrpic-doping concentrations, PFO emissions were completely quenched; with 8 wt $\%$ FIrpic, a maximum luminous efficiency of $11.5 \mathrm{~cd} / \mathrm{A}$ was achieved.
\end{abstract}

polymer light-emitting diode, phosphorescent, organic electroluminescence, back energy transfer

Citation: $\quad$ Niu Q L, Zhang Y, Wang Y L, et al. High-efficiency conjugated-polymer-hosted blue phosphorescent light-emitting diodes. Chin Sci Bull, 2012, 57: 3639-3643, doi: 10.1007/s11434-012-5247-3

Despite thirteen years of extensive research attention, phosphorescent organic/polymer light-emitting diodes (OLEDs/ PLEDs) still generate considerable interest as potential candidates for use in flat panel displays and solid-state lighting [1-5]. Either a small organic molecular material or a polymer can be adopted as a phosphorescent dye host [6]. Of these, polymer hosts are more promising for use in largesized flexible products due to the underlying solution processing technology $[7,8]$.

Non-conjugated polymer poly $(N$-vinylcarbazole) (PVK) has been a commonly used polymer host for phosphorescent dyes [9-12]. As a host polymer, PVK has however an inherent defect in that its electron and hole mobility difference is too large. To improve the electron mobility, PVK must be doped with an electron-transporting material [13]. However, in fabricating functional layer on top of the emissive layer (EML), electron-transporting material would be selectively removed resulting in poor luminescent efficiency $[14,15]$. For example, $\mathrm{Xu}$ et al. [14] found that the spin-

*Corresponding author (email: qlniu@ scnu.edu.cn) coating of the electron-injection layer from the methanol solution was capable of selectively extracting the electrontransporting material, 1,3-bis[2-(4-tert-butylphenyl)-1,3,4-oxadiazo-5-yl]benzene (OXD-7), of the EML, PVK:OXD-7: FIrpic:(piq) $)_{2} \operatorname{Ir}(\mathrm{acac})$, leading to poor PLED performance.

Poly(9,9-dioctylfluorene) (PFO) is a commonly-used blue material and host polymer for red phosphorescent dyes in PLEDs industry [16-19]. Previously, PFO was thought to be a bad choice as a host for blue or green phosphorescent dyes because of its low-lying triplet energy level. Although energy back transfer happened in both PL and EL processes, efficient green phosphorescent PLEDs were fabricated recently with a PFO host [20,21]. In contrast to PVK-hosted PLEDs, the performances of PFO-hosted PLEDs could be enhanced from the selective removal of the electron-transporting material during fabrication of the functional layer [22]. In this manner, PFO becomes a promising polymer host for phosphorescent dyes. Obtaining efficient blue phosphorescent PLEDs on a PFO host will not only offer greater selection of polymer host but extend the approaches towards generation of white light that would boost the application of 
phosphorescent PLEDs.

In this article, we report on the fabrication of high-efficiency blue phosphorescent EL with the conjugated-polymer PFO as a FIrpic host. The triplet energy level of PFO lies lower than that of FIrpic, causing energy back transfer from FIrpic to PFO that leads to poor PLEDs performances. However, by inserting PVK as a hole-transporting layer (HTL), the energy back transfer was suppressed because of the higher triplet energy level of PVK than FIrpic. At low FIrpic-doping concentrations, PFO was quenched completely. A luminous efficiency of $11.5 \mathrm{~cd} / \mathrm{A}$ was achieved, which was comparable with that of the PVK hosted PLED, $9.1 \mathrm{~cd} / \mathrm{A}$.

\section{Experimental}

The device structure consists of ITO/poly(3,4-ethylenedioxythiophene): poly(styrene sulfonate) (PEDOT:PSS) $(40 \mathrm{~nm}) /$ PVK (40 nm) /PFO:OXD-7 (30 wt $\%)$ : FIrpic $(x \mathrm{wt} \%)(70 \mathrm{~nm}) /$ $\mathrm{CsF}(1 \mathrm{~nm}) / \mathrm{Al}(100 \mathrm{~nm})$, where OXD-7 was incorporated to improve the electron current in the EML. The chemical structures of the relevant materials are shown in Scheme 1. Prior to device fabrication, an ITO-coated glass substrate was subjected to wet-cleaning in an ultrasonic bath sequentially filled with acetone, detergent, deionized water, and isopropanol. Next, the substrate was baked in a vacuum oven at $80^{\circ} \mathrm{C}$ for $2 \mathrm{~h}$. Before the coating with PEDOT: PSS, the substrate was treated for $10 \mathrm{~min}$ with $\mathrm{O}_{2}$ plasma. The $40 \mathrm{~nm}$ of PEDOT film (P4083) was spun-cast onto the ITO surface, and then baked in a vacuum oven at $90^{\circ} \mathrm{C}$ for $12 \mathrm{~h}$ to remove residual water. Subsequently, HTL and EML were sequentially spun-coated to a thickness of about 40 and $70 \mathrm{~nm}$, respectively, as determined by profilometry (XP-2). Finally, cathodes of CsF (1 nm) and then Al (200 nm) were thermally evaporated at a basic pressure of $3 \times 10^{-4} \mathrm{~Pa}$. The active emission area defined by a shadow mask was $0.15 \mathrm{~cm}^{2}$. Except for the coating process of the PEDOT layer, all processes were performed in a $\mathrm{N}_{2}$ atmosphere dry box (Mikrouna) with water and oxygen concentration less than

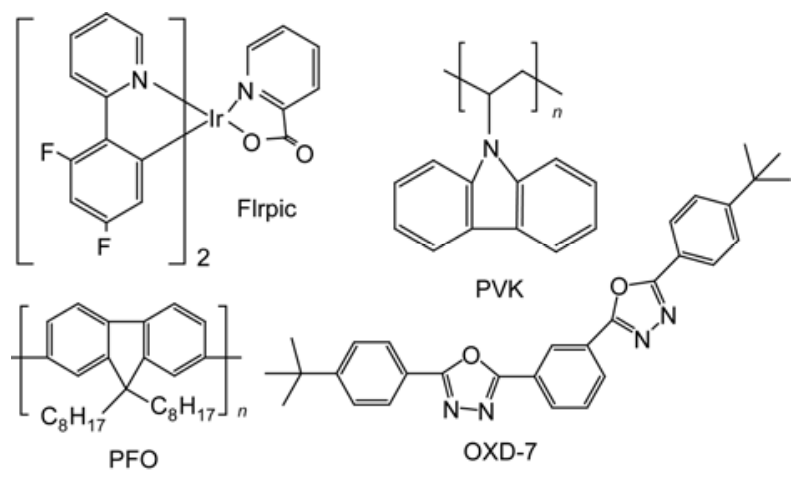

Scheme 1 The chemical structures of PFO, PVK, OXD-7 and FIrpic.
1 ppm. PVK-PLEDs doped with $30 \mathrm{wt} \%$ OXD-7 as host at $10 \mathrm{wt} \%$ FIrpic were also fabricated and tested under the same conditions.

Data of the current density, operating voltage, and luminous efficiency were recorded using a Keithley 2400 source measurement unit coupled with a calibrated silicon photodiode. Emission spectra were measured with a PR-705 SpectraScan Spectrophotometer (Photo Research).

\section{Results and discussion}

Figure 1 shows the PL spectra of various PFO thin films doped with FIrpic at different weight ratios: $0.1 \%, 1 \%, 8 \%$, $10 \%$ and $100 \%$. The PL spectrum of FIrpic peaked at 475 $\mathrm{nm}$. In the spectra of the blended thin films, the peak at 425 $\mathrm{nm}$ originated from PFO, and no FIrpic emission was observed. That is to say, FIrpic was completely quenched even at high doping concentrations. Similar quenching phenomena have been observed before for conjugated polymers $[20,21,23]$. Previous studies showed that the lower triplet energy level of the polymer host would cause energy back transfer from phosphorescent dye to polymer, leading to phosphorescent quenching [20,21,23-25]. A schematic diagram of the energy levels for PFO, FIrpic and PVK is displayed in Figure 2. Since the triplet energy level of PFO $(2.3 \mathrm{eV})$ [20] is lower than that of FIrpic (2.65 eV) [26], energy back transfer occurs from the triplet excitons on the FIrpic to the low-lying triplet states of PFO that causes the FIrpic quenching. Therefore, in the PL process, FIrpic was quenched by PFO because of the low-lying triplet energy level of PFO.

FIrpic quenching also occurs in the EL process, as inferred from the EL spectra of the devices without the PVK interlayer, ITO/PEDOT/PFO: OXD-7 (30 wt $\%$ ): FIrpic $(x \mathrm{wt} \%) / \mathrm{CsF} / \mathrm{Al}$, with FIrpic weight ratios varying from 0 , $0.1 \%, 1 \%, 8 \%, 10 \%$ to $16 \%$, as seen in Figure 3. With no more than $1 \mathrm{wt} \%$ FIrpic, only the PFO emission was observed,

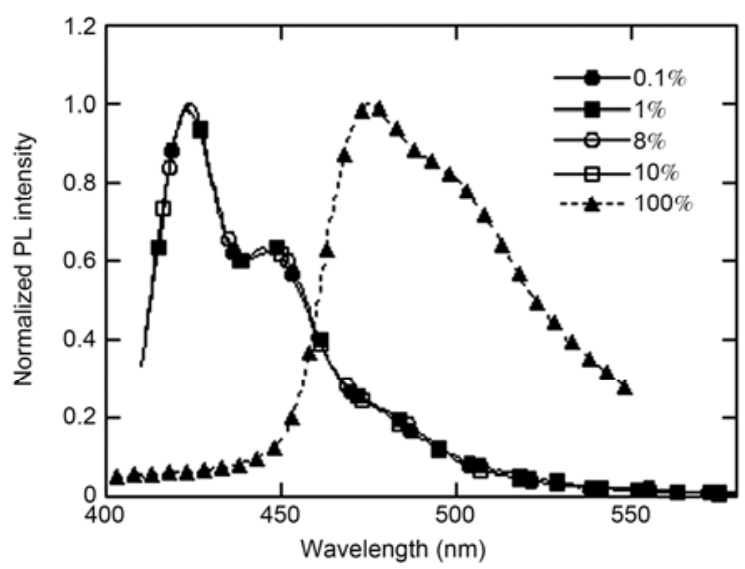

Figure 1 PL spectra of thin films, PFO doped with FIrpic at different weight ratios: $0.1 \%, 1 \%, 8 \%, 10 \%$ and $100 \%$. 

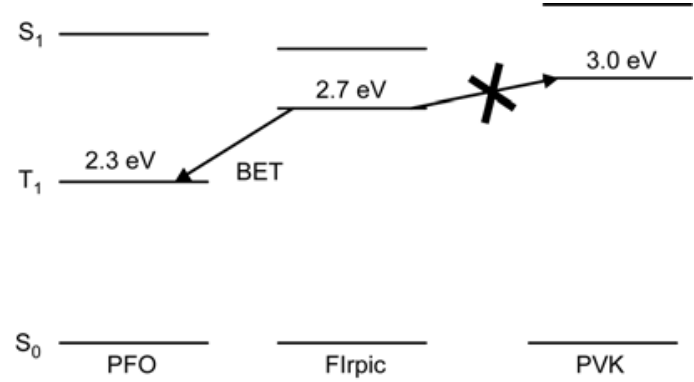

Figure 2 Schematic diagram of energy levels of PFO, FIrpic and PVK.

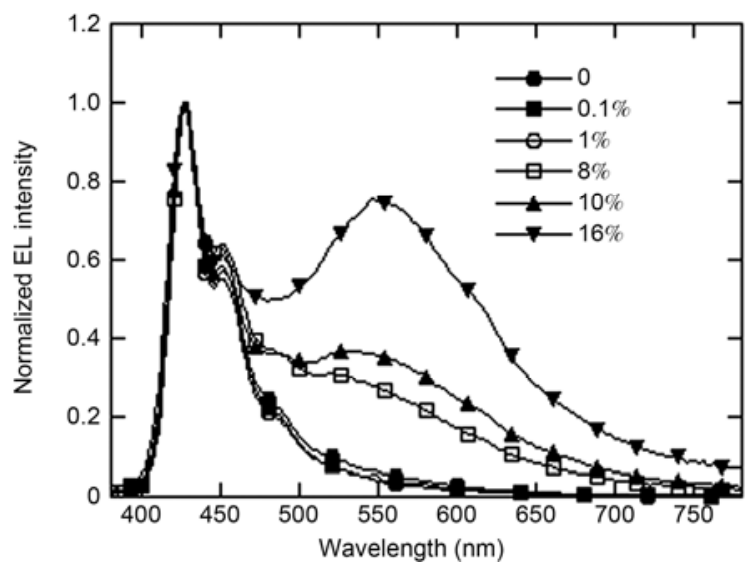

Figure 3 EL spectra of the devices without PVK layer, with FIrpic concentrations varying from $0,0.1 \%, 1 \%, 8 \%, 10 \%$ to $16 \%$.

the emission peak being located at $427 \mathrm{~nm}$, along with a shoulder peak at $450 \mathrm{~nm}$. In contrast to PL spectra, at high FIrpic concentrations of $8 \%, 10 \%$, and $16 \%$, FIrpic emissions were detected simultaneously with a peak at $547 \mathrm{~nm}$ that can be attributed to charge trapping of FIrpic in the EL process. Nevertheless, the relative intensity of FIrpic was much weaker than that of PFO, suggesting FIrpic quenching by PFO [20,21]. Therefore, in PFO matrix, FIrpic quenching has occurred in both PL and EL processes.

Device structures of PLEDs were modified by inserting

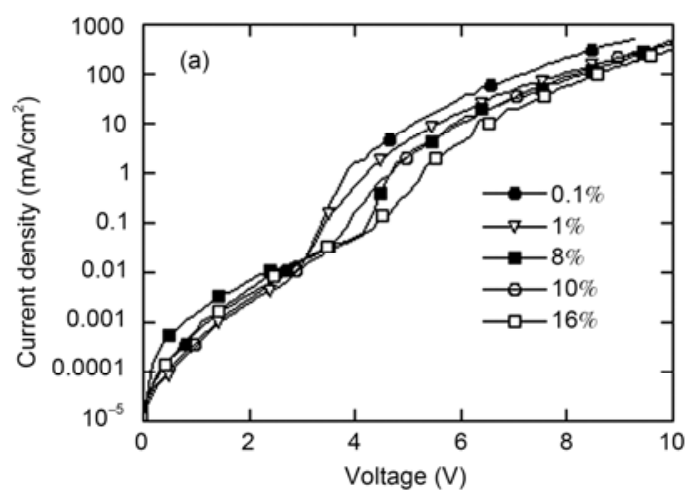

HTL PVK between PEDOT and the EML. Figure 4 shows the EL spectra of devices with PVK interlayer, ITO/PEDOT/ PVK/PFO: OXD-7 (30 wt $\%)$ : FIrpic $(x \mathrm{wt} \%) / \mathrm{CsF} / \mathrm{Al}$, with FIrpic weight ratios of varied from 0 to $16 \%$. With the exception of the PFO emission, another emission band was also observed clearly from FIrpic. Differing from the EL spectra of devices without PVK layer, here PFO was almost quenched at the low FIrpic concentration of $1 \mathrm{wt} \%$, indicating the PVK layer suppressed the energy back transfer. Similar to previous research of ours on the host-guest combination of PFO and $\operatorname{Ir}(\mathrm{ppy})_{3}$, exothermal energy-transfer quenching from the excited state of $\operatorname{Ir}(\mathrm{ppy})_{3}$ was circumvented by inserting a PVK interlayer [20,21]. According to previous studies, two features of PVK were responsible for the minimization of phosphorescent quenching, the high lowest unoccupied molecular orbit (LUMO) level, and the high triplet energy level $[20,21,27]$. Since the LUMO of PVK $(-2.0 \mathrm{eV})$ is much higher than that of PFO $(-2.4 \mathrm{eV})$, electrons can be blocked by PVK at the PVK-EML interface. Thus, the recombination zone is located at the PVK-EML interface and excitons form on PVK at the interface [21]. As seen in Figure 2, PVK has a triplet energy level of $3.0 \mathrm{eV}$

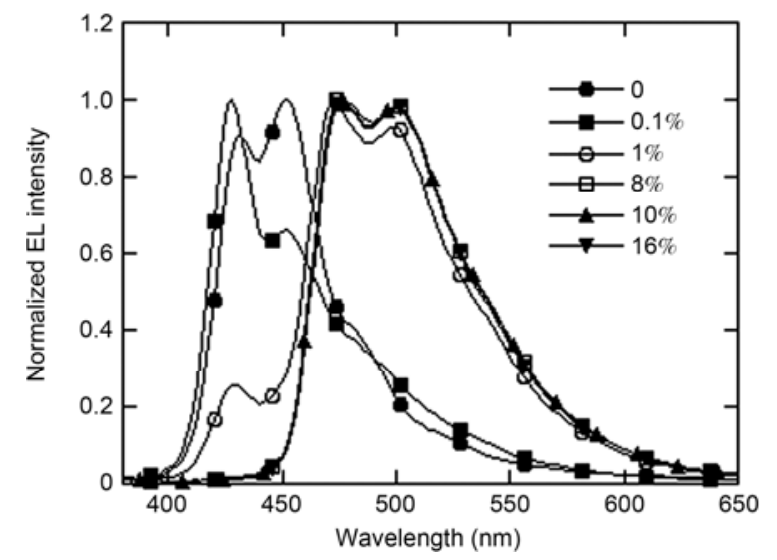

Figure 4 EL spectra of the devices with PVK layer, with FIrpic weight ratios varying from 0 to $16 \%$.

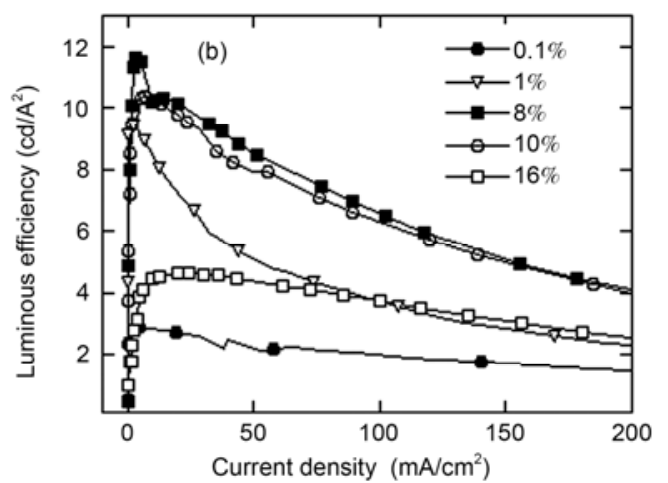

Figure 5 Characteristics of current density-voltage (a) and luminous efficiency-current density (b) of the PFO hosted devices at $0.1 \%$, $1 \%, 8 \%, 10 \%$, and $16 \%$ FIrpic. 


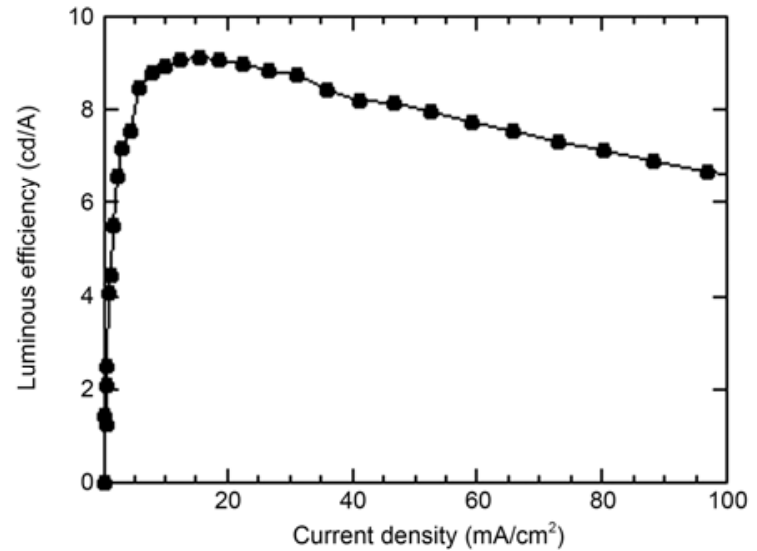

Figure 6 Luminous efficiency-current density characteristics of the PLED with PVK: OXD-7 (30 wt\%) doped with 10 wt\% FIrpic.

[27], which is much higher than the FIrpic level $(2.65 \mathrm{eV})$, energy back transfer from FIrpic to PVK would not occur. The PVK excitons can be readily transferred to FIrpic, via Förster or Dexter energy transfer, leading to efficient blue electrophosphorescence [27]. Thus, quenching of the excited state of FIrpic to the triplet state of PFO is circumvented.

Current density-voltage and luminous efficiency-current density characteristics of the PLEDs with PVK interlayer and various FIrpic concentrations are shown in Figure 5. The luminous efficiencies show strong FIrpic concentration dependence, that increased with increasing FIrpic concentration up to $8 \mathrm{wt} \%$ and then decreased. The optimum device showed a maximum luminous efficiency of $11.5 \mathrm{~cd} / \mathrm{A}$ with the 1931 CIE coordinates of $(0.18,0.41)$. PVK hosted PLED with the structure of ITO/PEDOT/PVK: OXD-7 (30 wt\%): FIrpic (10 wt $\%$ ) was tested [28], for which luminous efficiency-current density characteristic is shown in Figure 6. The maximum luminous efficiency was $9.1 \mathrm{~cd} / \mathrm{A}$, which was slightly lower than that of the PFO hosted device.

\section{Conclusion}

Highly-efficient blue phosphorescent PLEDs based on a PFO host were fabricated. Because of the low-lying triplet energy of PFO, energy back transfer occurred from FIrpic to PFO in both PL and EL processes. At high FIrpic-doping concentrations in PFO, only a weak emission from FIrpic was observed. By inserting a PVK interlayer, energy back transfer was suppressed; at low doping concentrations, PFO was completely quenched. A maximum luminous efficiency of $11.5 \mathrm{~cd} / \mathrm{A}$ was achieved with $8 \mathrm{wt} \%$ FIrpic.

This work was supported by the National Natural Science Foundation of China (10904042 and 61078046), the Key Progam of Chinese Ministry of Education (210157), China Postdoctoral Science Foundation (2012M511584), the Natural Science Foundation of Guangdong Province (8251063101000007 and 10151063101000009), the Scientific and Technological Plan of Guangdong Province (2008B010200004 and 2010B010600030) and the Scientific and Technological Plan of Guangzhou City (10U1-D00131).
1 Koo W H, Jeong S M, Araoka F, et al. Light extraction from organic light-emitting diodes enhanced by spontaneously formed buckles. Nat Photon, 2010, 4: 222-226

2 Sax S, Rugen-Penkalla N, Neuhold A, et al. Efficient blue-lightemitting polymer heterostructure devices: The fabrication of multilayer structures from orthogonal solvents. Adv Mater, 2010, 22: 2087-2091

3 Wang B Z, Zhang A Q, Wu H B, et al. Polymer white light-emitting diodes with a single emission layer of fluorescent polymer blend. Acta Phys Sin, 2010, 59: 4240

4 Zhu D X, Zhen H Y, Ye H, et al. Green polarized electroluminescence from poly (9,9-dioctylfluorene-alt-benzothiadiazole). Acta Phys Sin, 2009, 58: 2067

5 Bolink H J, Brine H, Coronado E, et al. Phosphorescent hybrid organi-inorganic light-emitting diodes. Adv Mater, 2010, 22: 2198

6 Sun Y R, Giebink N C, Kanno H, et al. Management of singlet and triplet excitons for efficient white organic light-emitting devices. Nature, 2006, 440: 908-912

7 Lee D H, Choi J S, Chae H, et al. Highly efficient phosphorescent polymer OLEDs fabricated by screen printing. Display, 2008, 29: 436-439

8 Gustafsson G, Cao Y, Treacy G. M, et al. Flexible light-emitting diodes made from soluble conducting polymers. Nature, 1992, 357: 477-479

9 Yook K S, Jang S E, Lee J Y. Efficiency improvement of solution processed blue phosphorescent devices using high triplet energy electron transport layer. Electrochem Solid-State Lett, 2010, 13: J122-J124

10 Li Y H, Fang Y A, Zou J H, et al. Efficient polymer white-lightemitting devices based on two complementary phosphorescent colors. Acta Phys-chim Sin, 2010, 26: 2752-2756

11 Ko L C, Liu T Y, Chen C Y, et al. Multi-layer organic light-emitting diodes processed from solution using phosphorescent dendrimers in a polymer host. Org Electron, 2010, 11: 1005-1009

$12 \mathrm{Wu} \mathrm{H} \mathrm{B}$, Zhou G J, Zou J H, et al. Efficient polymer white-lightemitting devices for solid-state lighting. Adv Mater, 2009, 21: 4181

13 Lee D H, Xun Z, Chae H, et al. Effect of electron- and hole-transporting materials on the performance of FIrpic-doped PVK phosphorescent devices. Synth Met, 2009, 159: 1640-1643

14 Xu Y, Yang R, Peng J, et al. Solvent effects on the architecture and performance of white-emitting polymer light-emitting diodes with conjugated oligoelectrolyte electron transport layers. Adv Mater, 2009, 21: $584-588$

15 Zhang Y, Huang F, Chi Y, et al. Highly efficient white polymer lightemitting diodes based on nanometer-scale control of the electron injection layer morphology through solvent processing. Adv Mater, 2008, 20: 1565-1570

16 Niu Q, Shao Y, Xu W, et al. Full color and monochrome passivematrix polymer light-emitting diodes flat panel displays made with solution processes. Org Electron, 2008, 9: 95-100

17 Liang B, Xu Y H, Chen Z, et al. White polymer phosphorescent lightemitting devices with a new yellow-emitting iridium complex doped into polyfluorene. Synth Met, 2009, 159: 1876-1879

$18 \mathrm{Wu}$ H, Ying L, Yang W, et al. Progress and perspective of polymer white light-emitting devices and materials. Chem Soc Rev, 2009, 38: 3391-3400

19 Xu Y H, Peng J B, Mo Y Q, et al. Efficient white-light-emitting diodes based on polymer co-doped with two phosphorescent dyes. Appl Phys Lett, 2005, 87: 193502

20 Niu Q, Zhang Y, Fan G. Highly efficient phosphorescence lightemitting diodes with conjugated polymer host. Acta Phys Sin, 2009, 58: 530-534

21 Chen Z, Jiang C, Niu Q, et al. Enhanced green electrophosphorescence by using polyfluorene host via interfacial energy transfer from polyvinylcarbazole. Org Electron, 2008, 9: 1002-1009

22 Niu Q, Wang X, Zhou J, et al. Effect of methanol treatment on performance of phosphorescent dye doped polymer light-emitting diodes. Synth Met, 2010, 160: 2381-2384

23 Zhang X, Jiang C, Mo Y, et al. High-efficiency blue light-emitting electrophosphorescent device with polymer host. Appl Phys Lett, 2006, 88: 051116 
24 Sudhakar M, Djurovich P I, Hogen-Esch T E. Phosphorescence uenching by conjugated polymers. J Am Chem Soc, 2003, 125: 7796

25 Chen F C, He G F, Yang Y. Triplet exciton confinement in phosphorescent polymer light-emitting diodes. Appl Phys Lett, 2003, 82: 1006

26 Lee S J, Seo J H, Kim G Y, et al. A study on the phosphorescent blue organic light-emitting diodes using various host materials. Mol Cryst
Liq Cryst, 2009, 507: 345

27 Li A, Li Y, Cai W, et al. Realization of highly efficient white polymer light-emitting devices via interfacial energy transfer from poly ( $N$-vinylcarbazole). Org Electron, 2010, 11: 529

28 Mathai M K, Choong V E, Choulis S A, et al. Highly efficient solution processed blue organic electrophosphorescence with $141 \mathrm{~m} / \mathrm{W}$ luminous efficacy. Appl Phys Lett, 2006, 88: 243512

Open Access This article is distributed under the terms of the Creative Commons Attribution License which permits any use, distribution, and reproduction in any medium, provided the original author(s) and source are credited. 